Symmetries, Differential Equations and Applications (SDEA-II)

International Journal of Modern Physics: Conference Series

Vol. 38 (2015) 1560075 (8 pages)

(C) The Authors

DOI: $10.1142 / \mathrm{S} 2010194515600757$

\title{
Lie symmetry analysis of some time fractional partial differential equations
}

\author{
E. H. El Kinani \\ A.A Group, Mathematical Department, Moulay Ismail University, \\ Faculty of Sciences and Technics Errachidia, BP 509, Morocco \\ Université Moulay Ismail Ecole Nationale Supérieure \\ des Arts et Métiers (ENSAM), Meknès, BP 15290 Al Mansour, Morocco \\ h.elkinani@ensam.umi.ac.ma
}

A. Ouhadan

Centre Régional des Métiers de l'Education et de la Formation

Meknès, BP 255, Morocco

aouhadan@gmail.com

Published 30 April 2015

\begin{abstract}
This paper uses Lie symmetry analysis to reduce the number of independent variables of time fractional partial differential equations. Then symmetry properties have been employed to construct some exact solutions.

Keywords: Lie symmetry method; fractional derivative; time fractional differential equation; similarity variables.
\end{abstract}

\section{Introduction}

In recent years, fractional differential equations attracted more attention as they are widely used to describe several phenomena in many fields of sciences such as fluid flow, biology, chemistry, control theory and other areas. ${ }^{1}$ Consequently, many powerful and efficient methods for seeking exact and numerical solutions have been developed including the Adomian's decomposition method, ${ }^{2}$ the variational iteration method, ${ }^{3,4}$ transformation methods, ${ }^{5}$ the finite difference method, ${ }^{6}$ the first integral method, ${ }^{7}$ and so on.

Lie symmetry analysis is an important method in studying differential equations with integer order. ${ }^{8-10}$ This method has several applications including construction of new solutions from trivial ones, possibility of some non linear equations to be linearized, construction of integrator factor, reduction of order, reduction of the

This is an Open Access article published by World Scientific Publishing Company. It is distributed under the terms of the Creative Commons Attribution 3.0 (CC-BY) License. Further distribution of this work is permitted, provided the original work is properly cited. 
number of independent variables and so on. Concerning symmetry analysis of fractional partial differential equations, the recognized results, in the literature, are very few and the method is still not sufficiently developed. Rigorous study of symmetries, admitted by such fractional equations, was started by Gazizov et al. in ${ }^{11}$ where they have used two basic fractional derivatives: Riemann-Liouville and Caputo.

We use Lie symmetries with the prolongation formula given by Gazizov et al. ${ }^{11}$ for the time fractional partial differential equation given by

$$
\frac{\partial^{\alpha} u}{\partial t^{\alpha}}=x \frac{\partial^{2} u}{\partial x^{2}}+f(x) \frac{\partial u}{\partial x},
$$

where $\frac{\partial^{\alpha} u}{\partial t^{\alpha}}$ is the fractional derivative of order $\alpha$ with $0<\alpha<1$. The above equation has been extensively studied in the case of $\alpha=1$ by Craddock $^{12}$ with different expressions of the drift function $f$ which satisfies a family of Riccati type differential equations. In this paper, we are interested in two cases for the drift function, the first is $f(x)=\beta \in \mathbb{R}$ and the second is $f(x)=\frac{1+3 \sqrt{x}}{2(1+\sqrt{x})}$.

There is no unique definition of fractional derivatives. ${ }^{13,14}$ Here we use the Riemann-Liouville version, defined by

$$
D^{\alpha} u(t, x)=\frac{\partial^{\alpha} u}{\partial t^{\alpha}}= \begin{cases}\frac{\partial^{n} u}{\partial t^{n}}, & \alpha=n \in \mathbb{N} \\ \frac{1}{\Gamma(n-\alpha)} \frac{\partial^{n}}{\partial t^{n}} \int_{0}^{t} \frac{u(v, x)}{(t-v)^{\alpha+1-n}} d v, & n-1<\alpha<n, n \in \mathbb{N}^{*},\end{cases}
$$

where $\Gamma$ is the well-known gamma function, and $D^{\alpha}$ satisfies

$$
\begin{aligned}
& D^{\alpha} t^{\gamma}=\frac{\Gamma(\gamma+1)}{\Gamma(\gamma+1-\alpha)} t^{\gamma-\alpha}, \alpha>0, \gamma>-1, t>0, \\
& D^{\alpha} 1=\frac{t^{-\alpha}}{\Gamma(1-\alpha)}, \alpha \geq 0, t>0 .
\end{aligned}
$$

This paper is organized as follows. In the second section, a background of symmetry analysis is given and the system of determining equations for symmetry generators corresponding to studied equation is obtained. In the third section, the obtained system of determining equations is solved and infinitesimal generators of each case are given. In the fourth section, some special exact solutions of Eq. (1) are pointed out. Finally, a conclusion is given.

\section{Symmetry Analysis of Fractional Partial Differential Equations}

Equation (1) is a time fractional partial differential equation of the form

$$
\frac{\partial^{\alpha} u}{\partial t^{\alpha}}=F\left(t, x, u, u_{x}, u_{x x}, \ldots\right), \quad 0<\alpha<1,
$$

where subscripts denote partial derivatives. Equation (1) is invariant under a one parameter continuous transformations: ${ }^{8,9}$

$$
\begin{aligned}
\widetilde{t} & =t+\varepsilon \tau(t, x, u)+o(\varepsilon), \\
\widetilde{x} & =x+\varepsilon \xi(t, x, u)+o(\varepsilon), \\
\widetilde{u} & =u+\varepsilon \varphi(t, x, u)+o(\varepsilon),
\end{aligned}
$$


with corresponding infinitesimal generator given by

$$
X=\tau(t, x, u) \frac{\partial}{\partial t}+\xi(t, x, u) \frac{\partial}{\partial x}+\varphi(t, x, u) \frac{\partial}{\partial u},
$$

where $\tau, \xi$ and $\varphi$ are infinitesimals to be determined later. To apply Lie's algorithm, we need to extend the infinitesimal generator $X$ to $X^{\alpha}$ of the form

$$
X^{\alpha}=X+\varphi^{x} \frac{\partial}{\partial u_{x}}+\varphi^{x x} \frac{\partial}{\partial u_{x x}}+\varphi^{\alpha} \frac{\partial}{\partial u^{\alpha}},
$$

such that $u^{\alpha}=\frac{\partial^{\alpha} u}{\partial t^{\alpha}}$ and $\varphi^{x}, \varphi^{x x}, \varphi^{\alpha}$ are extended infinitesimals of order 1,2 and $\alpha$ respectively. $\varphi^{x}$ and $\varphi^{x x}$ have the form

$$
\begin{aligned}
\varphi^{x}= & \varphi_{x}+\left(\varphi_{u}-\xi_{x}\right) u_{x}-\tau_{x} u_{t}-\xi_{u} u_{x}^{2}-\tau_{u} u_{x} u_{t}, \\
\varphi^{x x}= & \varphi_{x x}+\left(2 \varphi_{x u}-\xi_{x x}\right) u_{x}-\tau_{x x} u_{t}+\left(\varphi_{u u}-2 \xi_{x u}\right) u_{x}^{2}-2 \tau_{x u} u_{x} u_{t} \\
& -\xi_{u u} u_{x}^{3}-\tau_{u u} u_{x}^{2} u_{t}+\left(\varphi_{u}-2 \xi_{x}\right) u_{x x}-2 \tau_{x} u_{x t}-3 \xi_{u} u_{x} u_{x x} \\
& -\tau_{u} u_{x x} u_{t}-2 \tau_{u} u_{x} u_{x t} .
\end{aligned}
$$

Nevertheless, the $\alpha$-th extended infinitesimal $\varphi^{\alpha}$ related to Riemann-Liouville fractional time derivative has the following form

$$
\varphi^{\alpha}=D_{t}^{\alpha}(\varphi)+\xi D_{t}^{\alpha}\left(u_{x}\right)-D_{t}^{\alpha}\left(\xi u_{x}\right)+D_{t}^{\alpha}\left(D_{t}(\tau) u\right)-D_{t}^{\alpha+1}(\tau u)+\tau D_{t}^{\alpha+1}(u),
$$

where $D_{t}^{\alpha}$ denotes the total time fractional derivative. The $\alpha$-th extended infinitesimal $\varphi^{\alpha}$ can be rewritten as ${ }^{11,15,16}$

$$
\begin{aligned}
\varphi^{\alpha}= & \frac{\partial^{\alpha} \varphi}{\partial t^{\alpha}}+\left(\varphi_{u}-\alpha D_{t}(\tau)\right) \frac{\partial^{\alpha} u}{\partial t^{\alpha}}-u \frac{\partial^{\alpha} \varphi_{u}}{\partial t^{\alpha}}+\mu \\
& +\sum_{n=1}^{+\infty}\left[\left(\begin{array}{l}
\alpha \\
n
\end{array}\right) \frac{\partial^{n} \varphi_{u}}{\partial t^{n}}-\left(\begin{array}{c}
\alpha \\
n+1
\end{array}\right) D_{t}^{n+1}(\tau)\right] D_{t}^{\alpha-n}(u) \\
& -\sum_{n=1}^{+\infty}\left(\begin{array}{l}
\alpha \\
n
\end{array}\right) D_{t}^{n}(\xi) D_{t}^{\alpha-n}\left(u_{x}\right)
\end{aligned}
$$

where

$$
\begin{aligned}
\mu= & \sum_{n=2}^{+\infty} \sum_{m=2}^{n} \sum_{k=2}^{m} \sum_{r=0}^{k-1}\left(\begin{array}{l}
\alpha \\
n
\end{array}\right)\left(\begin{array}{l}
n \\
m
\end{array}\right)\left(\begin{array}{l}
k \\
r
\end{array}\right) \frac{1}{k !} \\
& \times \frac{t^{n-\alpha}}{\Gamma(n+1-\alpha)}[-u]^{r} \frac{\partial^{m}}{\partial t^{m}}\left(u^{k-r}\right) \frac{\partial^{n-m+k} \varphi}{\partial t^{n-m} \partial u^{k}} .
\end{aligned}
$$

In our case, the term $\mu$ vanishes as a consequence of $\varphi_{u u}=0$. Equation (1) is invariant under transformations (2) if and only if the invariance condition is satisfies,i.e

$$
\varphi^{\alpha}-\left(u_{x x}+f^{\prime}(x) u_{x}\right) \xi-f(x) \varphi^{x}-x \varphi^{x x}=0, \quad \text { whenever } \quad u^{\alpha}=x u_{x x}+f(x) u_{x},
$$

where $f$ is the drift function. To obtain general form of infinitesimals $\xi, \tau$ and $\varphi$, we need to substitute expressions (3),(4) and (5) into invariance condition (7). 
The equation depends on variables $u_{x}, u_{x x}, u_{t}, u_{x t}, \ldots$ and $D_{t}^{\alpha-n} u, D_{t}^{\alpha-n} u_{x}$ for $n=$ $1,2, \ldots$. Those variables are considered to be independent variables.

Splitting the defining Eq. (7) with respect to independents variables mentioned above leads to the system of infinitely equations

$$
\begin{aligned}
& \xi_{t}=\xi_{u}=\tau_{x}=\tau_{u}=\varphi_{u u}=0, \\
& x \varphi_{u}-\alpha x \tau_{t}-\xi-x\left(\varphi_{u}-2 \xi_{x}\right)=0, \\
& -\alpha f(x) \tau_{t}-f^{\prime}(x) \xi+f(x) \xi_{x}-x\left(2 \varphi_{x u}-\xi_{x x}\right)=0, \\
& \frac{\partial^{\alpha} \varphi}{\partial t^{\alpha}}-u \frac{\partial^{\alpha} \varphi_{u}}{\partial t^{\alpha}}-f(x) \varphi_{x}-x \varphi_{x x}=0, \\
& \left(\begin{array}{l}
\alpha \\
n
\end{array}\right) \frac{\partial^{n} \varphi_{u}}{\partial t^{n}}-\left(\begin{array}{c}
\alpha \\
n+1
\end{array}\right) D_{t}^{n+1} \tau, \quad n=0,1,2, \ldots
\end{aligned}
$$

The above system is known as the system of determining symmetries and solving it leads to infinitesimal generators of symmetries admitted by Eq. (1).

\section{Admitted Symmetries of Studied Equation}

Examining equations (8) and (9) in the above system, we readily obtain

$$
\xi(x)=a \alpha x+m \sqrt{x}, \quad \text { and } \quad \tau(t)=a t+b,
$$

with $a, b$ and $m$ are arbitrary constants. In virtue of $\varphi_{u u}=0, \varphi$ must be linear in $u$. Thus

$$
\varphi=g(t, x) u+h(t, x),
$$

for some functions $g(t, x)$ and $h(t, x)$. On the other hand, Eq. (12) requires that

$$
\frac{\partial \varphi_{u}}{\partial t}=0, \quad \text { then } \quad g=g(x)
$$

We substitute these in Eq. (11) to derive that the function $h$ is an arbitrary solution of the original fractional differential Eq. (1). Substituting of $\xi$ and $\tau$ their expressions into Eq. (10) leads to the equation

$$
g^{\prime}(x)=\frac{1}{4 x \sqrt{x}} m f(x)-\frac{1}{2}\left(a \alpha+\frac{\sqrt{x}}{x} m\right) f^{\prime}(x)-\frac{m}{8 x \sqrt{x}} .
$$

Differentiation of the Eq. (11) over $u$ leads to

$$
x g^{\prime \prime}(x)+f(x) g^{\prime}(x)=0 .
$$

Differentiation of Eq. (13) with respect the independent variable $x$ and substituting this into Eq. (14), leads

$$
-\frac{a \alpha}{2} x \frac{d}{d x} \mathfrak{L} f+16^{-1} m x^{-\frac{1}{2}}\left[3+8 \mathfrak{L} f-8 x \frac{d}{d x} \mathfrak{L} f\right]=0 .
$$

with $\mathfrak{L} f=x f^{\prime}-f+\frac{f^{2}}{2}$. Finally, the choice of a function $y(x)$ leads to a drift function $f$ as a solution of the Ricatti type equation $x f^{\prime}-f+\frac{f^{2}}{2}=y(x)$ and hence Eq. (15) determines $m$ and $a$. 
Case 1: In this first case, we consider the fractional differential equation

$$
\frac{\partial^{\alpha} u}{\partial t^{\alpha}}=x \frac{\partial^{2} u}{\partial x^{2}}+\beta \frac{\partial u}{\partial x},
$$

where $\beta$ is an arbitrary constant. In this case we have $x f^{\prime \prime}+f f^{\prime}=0$. Using Eqs. (15) and (13) we obtain

- Subcase $1 . \beta=\frac{1}{2}$. In this case a basis for Lie algebra of symmetries admitted by Eq. (16) is

$$
\begin{aligned}
& X_{1}=\alpha x \frac{\partial}{\partial x}+t \frac{\partial}{\partial t}, \quad X_{2}=\sqrt{x} \frac{\partial}{\partial x} \\
& X_{3}=\frac{\partial}{\partial t}, \quad X_{4}=u \frac{\partial}{\partial u}, \quad X_{h}=h(t, x) \frac{\partial}{\partial u},
\end{aligned}
$$

with $h$ an arbitrary solution of Eq. (16).

- Subcase 2 . $\beta=\frac{3}{2}$, for this choice of $\beta$, the symmetry Lie algebra is spanned by $X_{1}, X_{3}, X_{4}, X_{h}$ and the infinitesimal generator $X_{5}$ given by

$$
X_{5}=\sqrt{x} \frac{\partial}{\partial x}-\frac{1}{2 \sqrt{x}} u \frac{\partial}{\partial u} .
$$

- Subcase 3. Therefore, if the drift does not belongs to $\left\{\frac{1}{2}, \frac{3}{2}\right\}$ the only possible symmetries are $X_{1}, X_{3}, X_{4}$ and $X_{h}$.

Case 2: $f(x)=\frac{1+3 \sqrt{x}}{2(1+\sqrt{x})}$. In this case the drift function $f$ satisfies the Ricatti equation $x f^{\prime}-f+\frac{1}{2} f^{2}=-\frac{3}{8}$ and the equation arising from $f$ is

$$
\frac{\partial^{\alpha} u}{\partial t^{\alpha}}=x \frac{\partial^{2} u}{\partial x^{2}}+\frac{1+3 \sqrt{x}}{2(1+\sqrt{x})} \frac{\partial u}{\partial x} .
$$

Thus from Eq. (15) we obtain that $a$ and $m$ are arbitrary. Finally, the symmetry algebra admitted by Eq. (17) is spanned by $X_{3}, X_{4}, X_{h}$ and the infinitesimal generators $X_{6}$ and $X_{7}$ given by

$$
\begin{aligned}
& X_{6}=\alpha x \frac{\partial}{\partial x}+t \frac{\partial}{\partial t}+\frac{\alpha}{2(1+\sqrt{x})} u \frac{\partial}{\partial u}, \\
& X_{7}=\sqrt{x} \frac{\partial}{\partial x}-\frac{1}{2(1+\sqrt{x})} u \frac{\partial}{\partial u} .
\end{aligned}
$$

\section{Exact Solutions}

In this section we use the obtained symmetries to construct exact solutions.

\subsection{Reduction in the case $f(x)=\frac{1}{2}$ with $X_{2}+X_{4}$}

Invariants of the operator $X_{2}+X_{4}$ are $z=t, \quad$ and $\quad I=u e^{-2 \sqrt{x}}$. The corresponding invariant solution in this case has the form $u(t, x)=\psi(t) e^{2 \sqrt{x}}$, and the reduced 
equation is

$$
D_{t}^{\alpha}(\psi(t))=\psi(t)
$$

Let us consider that the above equation is a fractional order differential equation of order $\frac{2}{q}$ with $q$ a positive integer greater than 2 . Then a solution of the above reduced equation is given by ${ }^{14}$

$$
\psi(t)=\frac{1}{2} \sum_{k=0}^{q-1}\left\{E_{t}(-k \alpha, 1)-(-1)^{q-k-1} E_{t}\left(-k \alpha,(-1)^{q}\right)\right\},
$$

where the function $E_{t}$ is defined by

$$
E_{t}(\nu, a)=t^{\nu} \sum_{k=0}^{\infty} \frac{(a t)^{k}}{\Gamma(\nu+1+k)} .
$$

Consequently, the exact solution of original equation in this case is given by

$$
u(t, x)=\frac{1}{2} e^{2 \sqrt{x}} \sum_{k=0}^{q-1}\left\{E_{t}\left(-k \frac{\alpha}{2}, 1\right)-(-1)^{q-k-1} E_{t}\left(-k \frac{\alpha}{2},(-1)^{q}\right)\right\} .
$$

\subsection{Reduction in the case $f(x)=\frac{3}{2}$ with $X_{5}$}

The expression of this operator is

$$
X_{5}=\sqrt{x} \frac{\partial}{\partial x}-\frac{1}{2 \sqrt{x}} u \frac{\partial}{\partial u} .
$$

The corresponding invariants of $X_{5}$ are

$$
z=t, \quad \text { and } \quad I=u x^{\frac{1}{2}} .
$$

with invariant solution of the form

$$
u(t, x)=x^{-\frac{1}{2}} \psi(t)
$$

and the reduced equation is

$$
D_{t}^{\alpha}(\psi(z))=0 .
$$

Thus the invariant solution becomes

$$
u(t, x)=c x^{-\frac{1}{2}} t^{\alpha-1},
$$

with $c$ an arbitrary constant.

\subsection{Reduction in the case $f(x)=\frac{1+3 \sqrt{x}}{2(1+\sqrt{x})}$ with $X_{7}$}

This operator has the form $X_{7}=\sqrt{x} \frac{\partial}{\partial x}-\frac{1}{2(1+\sqrt{x})} u \frac{\partial}{\partial u}$. Corresponding invariants of $X_{8}$ are $z=t$, and $I=u \frac{1}{1+\sqrt{x}}=\psi(z)$. Invariant solution in this case has the form

$$
u(t, x)=\frac{1}{1+\sqrt{x}} \psi(t),
$$


and the reduced equation of (17) is

$$
D_{t}^{\alpha} \psi(t)=0
$$

The exact solution constructed in this case is given by

$$
u(t, x)=\frac{c}{1+\sqrt{x}} t^{\alpha-1},
$$

where $c$ is an arbitrary constant.

Remark 4.1. To obtain the group transformation generated by infinitesimal generator $X_{6}$, we solve the system of first order ordinary differential equations,

$$
\begin{aligned}
& \frac{d \widetilde{t}}{d \varepsilon}=\xi(\widetilde{t}, \widetilde{x}, \widetilde{u}), \\
& \frac{d \widetilde{x}}{d \varepsilon}=\varphi(\widetilde{t}, \widetilde{x}, \widetilde{u}), \\
& \frac{d \widetilde{u}}{d \varepsilon}=\varphi(\widetilde{t}, \widetilde{x}, \widetilde{u}),
\end{aligned}
$$

subject to the initial conditions

$$
\widetilde{t}(0)=t, \quad \widetilde{x}(0)=x, \quad \widetilde{u}(0)=u .
$$

The one-parameter groups $G$ generated by $X_{6}$ is given as follows. ${ }^{8}$ The entries gives the transformed point $\exp \left(\varepsilon X_{6}\right)(t, x, u)=(\widetilde{t}, \widetilde{x}, \widetilde{u})$ :

$$
G:\left(x e^{\alpha \varepsilon}, t e^{\varepsilon}, \frac{1+\sqrt{x}}{\sqrt{x}+e^{-\frac{\alpha}{2} \varepsilon}} u\right) .
$$

As $G$ is a symmetry group, so if $u(t, x)$ is a solution of equation (1), then is the transformed functions $\varepsilon . u(t, x)$ which is given by

$$
\varepsilon . u(t, x)=\frac{\sqrt{x}+e^{\frac{\alpha}{2} \varepsilon}}{\sqrt{x}+1} u\left(e^{-\varepsilon} t, e^{-\alpha \varepsilon} x\right),
$$

where $\varepsilon$ is sufficiently small real number. Using this invariance property, we can derive new exact solutions of equation (1) from the known one.

\section{Conclusion}

Using Lie point symmetry analysis we see that the studied time fractional partial differential equation can be transformed into fractional differential equations with a single independent variable. Consequently, some exact solutions are found and an example of a one parameter group of transformations is constructed.

\section{Acknowledgments}

The first author E. H. El Kinani would like to express his thanks to the organizers of SDEA-II Jan $27^{\text {th }}$-Jan $30^{\text {th }}$ (2014) in Islamabad Pakistan for their warm hospitality during the conference. Special thanks to Dr. Sajid Ali for his cooperation. Our thanks also to the anonymous referees for their careful reading of our manuscript. 


\section{References}

1. L. Debnath, Recent applications of fractional calculus to science and engineering, International Journal of Mathematics and Mathematical Sciences, Vol. 54, 3413-3442, 2003.

2. A. M. Wazwaz, S.M. El-Sayed, A new modification of the Adomian decomposition method for linear and nonlinear operators, Appl. Math. Comput. Vol. 122(2001)No.3, pp.393-405.

3. C. C. Wu, A fractional variational iteration method for solving fractional nonlinear differential equations, Comput. Math. Appl., vol.61, pp. 2186-2190, 2011.

4. J. Ji, J. B. Zhang and Y. J. Dong, The fractional variational iteration method improved with the Adomian series, Appl. Math. Lett., vol. 25, pp. 2223-2226, 2012.

5. B. Lu., Bäcklund transformation of fractional Riccati equation and its applications to nonlinear fractional partial differentialequations, Phys. Lett. A, vol. 376, pp. 20452048, 2012.

6. M.M. Meerschaert, H.P. Scheffler, C. Tadjeran., Finite difference methods for twodimensional fractional dispersion equation, J. Comput. Phys. 211(2006) 249-261.

7. M. Eslami, B. Fathi Vajargah, M. Mirzazadeh and Anjan Biswas, Application of first integral method to fractional partial differential equations, Indian Journal of Physics. Volume 88, Issue 2, 177-184, (2014).

8. P.J. Olver, Application of Lie groups to Differential Equation. New York: Springer; 1986.

9. G. Bluman, S. Kumei, Symmetries and Differential Equations. Applied Mathematical Sciences Series. 81 (Second ed.). New York: Springer-Verlag; 1989.

10. G.W. Bluman and J.D. Cole, The general similarity solutions of the heat equation, Journal of Mathematics and Mechanics, vol. 18(1969), pp. 1025-1042.

11. R.K. Gazizov, A.A. Kasatkin, S.Yu. Lukashchuk, Continuous transformation groups of fractional differential equations, Vestnik, USATU 9 (2007),pp. 125-135.

12. M. Craddock, E. Platen, Symmetry group methods for fundamental solutions, J. Differential Equations 207 (2004),pp. 285-302.

13. K.B. Oldham, J. Spanier, The fractional calculus.-Academic Press, 1974.-234p.

14. K.S. Miller, B. Ross, An introduction to the fractional calculus and fractional differential equations.-John Wiley and Sons, Inc; 1921.

15. R.K. Gazizov, A.A. Kasatkin, S.Yu. Lukashchuk, Symmetry properties of fractional diffusion equations, Phys. Scr. T136 (2009), 014-016.

16. E. Buckwar, Y. Luchko, Invariance of a partial differential equation of fractional order under the Lie group of scaling transformations, J. Math. Anal.Appl. 227 (1998). pp. 81-97.

17. S. Momani, Z. Odibat, Analytical solution of a time fractional NavierStokes equation by Adomian decomposition method, Appl. Math. Comput. 177(2006),pp. 488-494.

18. R. Hilfer, Applications of Fractional Calculus in Physics, Singapore: World Scientific; 2000.

19. I. Podlubny, Fractional differential equations: an introduction to fractional derivatives, fractional differential equations, to methods of their solution and some of their applications. New York: Academic Press; 1999. 